\title{
Bare Surface Soil Moisture Estimation Using Double-Angle and Dual-Polarization L-Band Radar Data
}

\author{
Xinyi Shen, Member, IEEE, Kebiao Mao, Qiming Qin, Yang Hong, and Guifu Zhang, Senior Member, IEEE
}

\begin{abstract}
Based on today's most widely used surface scattering model, the advanced integral equation model (AIEM), this study proposes a novel soil moisture inversion model that estimates bare surface soil moisture using double-incidence angle and dualpolarized L-band radar data. Compared with previous studies at L-/C-band, the proposed method provides the estimation of soil moisture without referring to the measured soil roughness and eliminates the requirement of an initial dry season condition. The root-mean-square error (rmse) of volumetric soil moisture varies from $0.8 \%$ to $3.2 \%$ at different incidence-angle combinations validated by simulated solving and from $4.0 \%$ to $7.9 \%$ by field measurements when the paired incidence angles are not both large. In case the paired angels are both large, not particularly suitable for soil moisture estimation, the rmse increases to $10.3 \%$. Therefore, this method is applicable to bare surface soil moisture retrieval when at least one of the incidence angles is not large.
\end{abstract}

Index Terms-Advanced integral equation model (IEM) (AIEM), double angle, dual polarization, microwave remote sensing, soil moisture inversion.

\section{INTRODUCTION}

$\mathbf{E}$ STIMATION and characterization of surface soil moisture's spatiotemporal variability are crucial in hydrologic modeling and water resource management. Remote sensing techniques are expected to contribute to such research topics in large scale. Among a variety of remote sensing methods, radar backscattering models that relate the soil dielectric constant, which is sensitive to $0 \%-35 \%$ moisture content [1], [2], are usually more reliable than other methods. During the past decades, a lot of effort has been made to derive electromagnetic

Manuscript received February 22, 2011; revised November 15, 2011 and August 31, 2012; accepted October 24, 2012. Date of publication February 26, 2013; date of current version June 20, 2013. This work was supported in part by the U.S. Department of Commerce under Grant BS123456.

X. Shen and Q. Qin are with the Institute of Remote Sensing and GIS, Peking University, Beijing 100871, China (e-mail: nerva_trajanvs@163.com; qmqinpku@163.com).

K. Mao is with the Key Laboratory of Resources Remote Sensing and Digital Agriculture, Ministry of Agriculture, Institute of Agricultural Resources and Regional Planning, Chinese Academy of Agricultural Sciences, Beijing 100081, China, and also with Shaanxi Normal University, Xi' an 710062, China (e-mail: kebiaomao2004@ hotmail.com).

Y. Hong and G. Zhang are with the School of Civil Engineering and Environmental Sciences, The University of Oklahoma, Norman, OK 73019 USA (e-mail: yanghong@ou.edu; guzhang1@ou.edu).

Color versions of one or more of the figures in this paper are available online at http://ieeexplore.ieee.org.

Digital Object Identifier 10.1109/TGRS.2012.2228209 wave scattering and emission models. The integral equation model (IEM) [3] is the most widely used theoretical radar scattering model for bare surface or sparsely vegetated landscapes. Mametsa et al. [4] concluded that the IEM had a much wider applicable region compared to the Kirchhoff approximation (KA) [5], [6] and the small perturbation model (SPM) [5], [6]. Due to its mathematical simplification and physical assumptions, IEM has been improved for many times [7], [8]. As the most updated version, advanced IEM (AIEM) [9], [10] writes the like-polarized formula in its compact form

$$
\begin{aligned}
\sigma_{p p}^{s}=\frac{k^{2}}{2} & \exp \left[-\sigma^{2}\left(k_{i z}^{2}+k_{s z}^{2}\right)\right] \\
& \times \sum_{n=1}^{\infty} \frac{\sigma}{n !}\left|I_{p p}^{n}\right|^{2} W^{(n)}\left(k_{s x}-k_{i x}, k_{s y}-k_{i y}\right) .
\end{aligned}
$$

If the surface is considered to be isotropic and exponentially correlated, AIEM is written as

$$
\sigma_{q p}^{0}=\operatorname{AIEM}\left(\varepsilon_{r}, h_{\mathrm{rms}}, L_{C}\right)
$$

where $h_{\mathrm{rms}}$ and $L_{C}$ stand for root mean square of surface height and the correlation length, respectively, and $\varepsilon_{r}$ is the relative dielectric constant of soil. The normalized radar cross section (NRCS) is a function of soil dielectric constant, surface correlation length, and standard deviation of height for any given frequency and incidence angle.

Compared with the time series algorithms [11]-[13] which require a priori knowledge, the so-called snapshot estimation methods that use only data of one frame or approximately spontaneity are of more challenge. The snapshot mode of soil moisture inversion is problematic because the radar backscattering is sensitive to both soil roughness and soil dielectric [14], [15], despite the acceptable accuracy of AIEM/IEM. To estimate soil moisture with various roughness, empirical [16], [17] and possibilistic [18] approaches were presented. Based on AIEM/IEM simulation, more quantitative inversion algorithms [19]-[24] have been developed. Compared with the empirical or possibilistic ones that require field measurements to fit their coefficients and, thus, potentially less applicable to other fields, this class has the merit of local independence. In bare soil cases, moisture and soil roughness are the three major independent parameters to determine. Considering the weak return of cross polarization compared with crosstalk and system noise [16], 
we only employ two copolarization measurements $\sigma_{\text {hh }}^{0}$ and $\sigma_{v v}^{0}$. To solve for soil moisture, we have to either decrease the unknowns or increase the measurements. By AIEM simulation, for natural surfaces, the value and dynamic range of copolarization ratio decrease with frequency. In addition, at C-band, they are even smaller than the noise level. In other words, $\sigma_{\mathrm{hh}}^{0}$ and $\sigma_{v v}^{0}$ approach each other too much at C-band. Therefore, one incidence angle can only provide one equation for inversion. Adding another incidence angle [24] introduces the difference (in decibels) $\Delta \sigma^{0}$ of two different incidence angles' backscattering to establish an extra equation of soil roughness named Z-index, i.e., $Z s=\left(h_{\mathrm{rms}}^{2} / L_{C}\right)$

$$
\Delta \sigma^{0}=f_{Z s}(Z s)
$$

To decrease the unknowns, [25] tried to combine the two soil $\left(h_{\mathrm{rms}}, L_{C}\right)$ roughness parameters into one unknown $Z s$ in the description of both $\sigma_{\mathrm{hh}}^{0}$ and $\sigma_{v v}^{0}$ at C-band which is valid when the product of wavenumber and $h_{\mathrm{rms}}$ is less than 1.2. To give a solution to a wider surface roughness region, [22] uses the dry season backscattering $\Delta \sigma_{\text {dry }}^{0}$ to establish another equation

$$
\sigma_{\mathrm{dry}}^{0}=f_{\mathrm{dry}}\left(h_{\mathrm{rms}}, L_{C}\right) .
$$

Since the soil moisture was set a constant 0.03 in this equation, $\Delta \sigma_{\text {dry }}^{0}$ was exclusively dependent on $h_{\mathrm{rms}}$ and $L_{C}$. By combining $\Delta \sigma^{0}$ and $\Delta \sigma_{\text {dry }}^{0}$ and then solving the nonlinear equation set, they mapped the soil roughness and input them to another prefitted four-order polynomial using either $\sigma_{\mathrm{hh}}^{0}$ or $\sigma_{v v}^{0}$ to finally estimate soil moisture

$$
m_{v}=f\left(\sigma_{p p}^{0}, h_{\mathrm{rms}}, L c\right) .
$$

Reference [19] employed six channels (three bands of two polarizations for each) to solve for soil moisture and roughness.

Progress made by the previous soil moisture inversion studies enables us to estimate soil moisture from remote sensing data. However, many physical limitations and mathematical problems still remain. Physically, the dry season requirement is often too strict to be applied because even a 5\% variation of soil moisture can spoil the fitting of $\sigma_{\mathrm{dry}}^{0}$, which is common even in dry fields; different bands $(\mathrm{L} / \mathrm{C} / \mathrm{X})$ are responsible for the moisture of different effective (penetrating) depths [26] and the roughness of different scales [27]; therefore, the unknowns are not identical in the equations at different bands. Mathematically, AIEM/IEM is a complicated and highly nonlinear formula that requires summing a large number of series. Direct use of it in the iterations of solving a nonlinear equation set would require considerable computation. Furthermore, by AIEM simulation, copolarizations of an individual band are highly correlated. Therefore, repeatedly using (12) to construct the equation set would be redundant.

This paper proposes an innovative soil moisture inversion model that, based on the latest AIEM, uses double-angle copolarized L-band radar data. The double incidence is generally attainable from the airborne synthetic aperture radar (SAR) and scatterometer data by repeating the flight swath, while constant soil moisture of the same area is assumed valid for consecutive satellite observations. Merits of this method enable us to do the following: 1) to avoid using prior information of soil roughness measurement; 2) to eliminate the dry season acquisition requirement; and 3) to implement a solving strategy that rapidly converges through the iterations. This paper is organized as follows. In Section II, an equation-set approach is presented and followed by the derivation of its three equations; a multiplicative method is proposed to relate copolarization measurements to soil parameters, a double-angle difference equation, and a copolarization ratio equation. The three equations are then combined to solve for the three soil parameters (dielectric factor, $h_{\mathrm{rms}}$ and $L_{C}$ ). Then, the solved dielectric factor is converted to volumetric soil moisture in the end of Section II. In Section III, a numerical solving strategy that uses a lookup table (LUT) to give an initial guess to solve the proposed equation set is described in greater detail. Section IV describes the experimental data [20], validation results, and error analysis, followed by the conclusion and discussion section at the end.

\section{MOdEL}

\section{A. Equation-Set Approach}

An equation-set approach is proposed to solve for the soil moisture in this study. This approach uses a number of equations that link radar measurements to the ground unknowns. To derive those equations, AIEM, the most widely acknowledged electromagnetic scattering model for random rough surface, is applied to provide a simulation database since it has the widest valid soil roughness range for natural surfaces and has been validated by many studies (see [4, Fig. 2]). The good accuracy of AIEM is also verified by using the field data introduced in Section IV.

However, the calculation of AIEM might be unnecessarily complex if used in equation-solving iterations. Fitting the AIEM simulation data to simpler equations is usually employed to circumvent this disadvantage. The range of simulation settings in this study is as follows: $m_{v} \in[0.01,0.4] \mathrm{cm}^{3} / \mathrm{cm}^{3}$ with $0.01 \mathrm{~cm}^{3} / \mathrm{cm}^{3}$ intervals, $L_{C} \in[1,30] \mathrm{cm}$ with 1 -cm intervals, and $h_{\mathrm{rms}} \in[0.1,3.5] \mathrm{cm}$ with $0.1-\mathrm{cm}$ intervals. At L-band, $\varepsilon_{r}$ is calculated empirically by [28] and adjusted by [29]. An exponential autocorrelation function is assumed throughout the study. This assumption is based on the fact that Gaussian 1.5 power and exponential functions were tested for the experimental data and the exponential one worked the best of all. By fitting different simulated radar measurements, a number of equations can be obtained.

Another issue in the equation-set approach is to avoid using equivalent equations in the equation set. The equivalence of a nonlinear equation set might be roughly evaluated by the correlation values between the measurements used by the equations. Through simulation, we found that the correlation values between $\sigma_{\text {hh }}^{0}$ and $\sigma_{v v}^{0}$ (in decibels) were above 0.99 for all incidence angles. Even between different incidence angles, the correlation is quite high. Meanwhile, our experimental data exhibit the corresponding properties. The correlation values between 


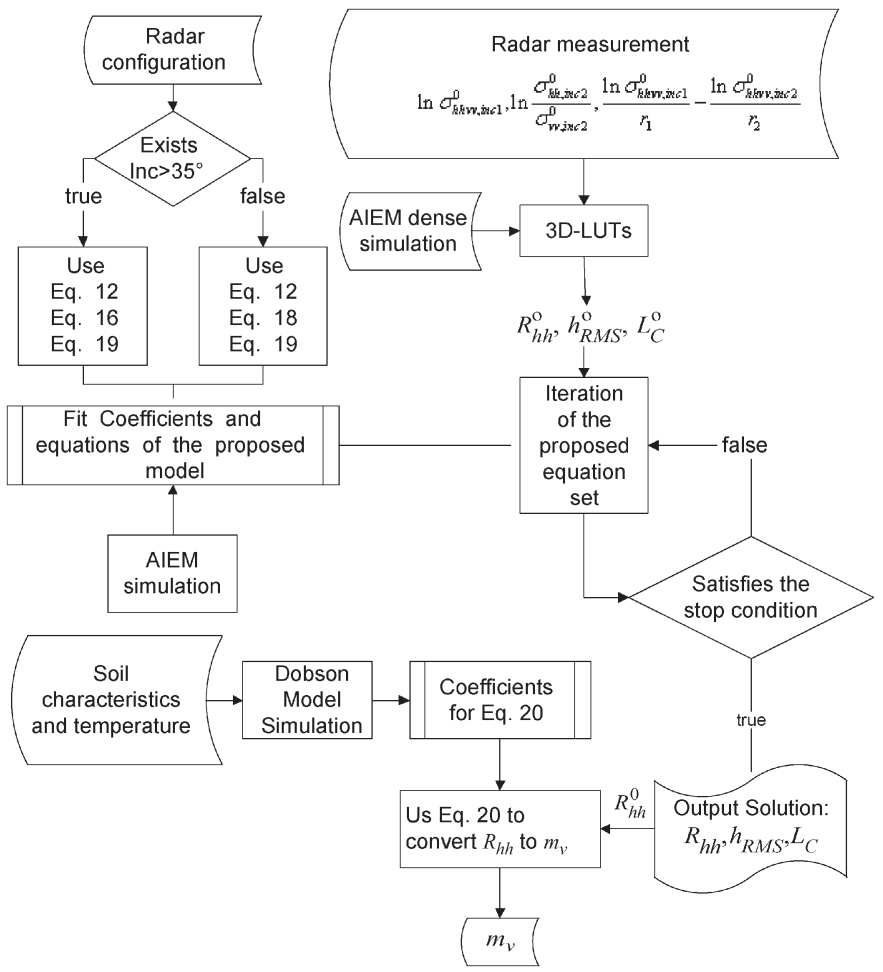

Fig. 1. Scheme of the proposed method.

$\sigma_{\mathrm{hh}}^{0}$ and $\sigma_{v v}^{0}$ (in decibels) are 0.9915/0.9940/0.9784/0.9792 for $20^{\circ} / 30^{\circ} / 40^{\circ} / 50^{\circ}$, respectively. For cross incidence angles, the correlation values are $0.9805 / 0.9736 / 0.9491 / 0.9920 /$ $0.9779 / 0.9857$ for $20^{\circ}$ versus $30^{\circ} / 20^{\circ}$ versus $40^{\circ} / 20^{\circ}$ versus $50^{\circ} / 30^{\circ}$ versus $40^{\circ} / 30^{\circ}$ versus $50^{\circ} / 40^{\circ}$ versus $50^{\circ} \mathrm{HH}$ polarization and $0.9817 / 0.9759 / 0.9541 / 0.9927 / 0.9814 / 0.9845$ for the same angle combinations at VV polarization. Therefore, we do not recommend the direct use of more than one $\sigma_{\mathrm{pp}}^{0}$ or their sum, arithmetic/geometric average to construct the equation set. Instead, their difference/ratio across polarization/incidence angle that are less correlated should be used. By carefully selecting the measurements with low correlation values, we found that, when the three measurements $\ln \sigma_{\text {hhvv }}^{0}, \Delta \ln \sigma_{\text {hhvv }}^{0}$, and $\ln \left(\sigma_{\mathrm{hh}}^{0} / \sigma_{\mathrm{vv}}^{0}\right)$ are used together, the equation set can be solved. The equations of these three measurements are derived through Sections II-C-E. They are (12), (16)/(18), and (19), respectively. In those equations, the three unknowns $R_{\mathrm{hh}}, h_{\mathrm{rms}}$, and $L_{C}$ are nonlinear coupled; thus, the proposed equation set is nonlinear which requires iterative solving. To accelerate the iterations and avoid the solutions from falling into a local optimum, a 3-D LUT is employed before iterations begin. Fig. 1 shows the scheme of solving, the details of which are described in Section III.

\section{B. Model at L-band}

Although L-band approaches the low-frequency region for natural surfaces more than C-band, SPM is not applicable to L-band land applications. Some studies [23] assume that

$$
\sigma_{\mathrm{pp}}^{0}=R_{\mathrm{pp}} S_{r}
$$

where $S_{r}$ stands for the roughness factor for both copolarizations and $R_{\mathrm{pp}}$ stands for the power of polarization amplitude in SPM and is written as

$$
\begin{aligned}
& R_{\mathrm{hh}}=\left|\alpha_{\mathrm{hh}}\right|^{2}=\left|\frac{\varepsilon_{r}-1}{\left(\cos \theta+\sqrt{\varepsilon_{r}-\sin ^{2} \theta}\right)^{2}}\right|^{2} \\
& R_{\mathrm{vv}}=\left|\alpha_{\mathrm{vv}}\right|^{2}=\left|\frac{\left(\varepsilon_{r}-1\right)\left[\sin ^{2} \theta-\varepsilon_{r}\left(1+\sin ^{2} \theta\right)\right]}{\left(\varepsilon_{r} \cos \theta+\sqrt{\varepsilon_{r}-\sin ^{2} \theta}\right)^{2}}\right|^{2}
\end{aligned}
$$

where $\theta$ denotes the incidence angle.

Through simulation, we found that, in the presence of very small $h_{\mathrm{rms}}$ or of small incidence angles $\left(\theta \leq 30^{\circ}\right)$, (6) did not introduce considerable error. In cases of both relatively large incidence angle $\left(\theta \leq 30^{\circ}\right)$ and large soil roughness $\left(h_{\mathrm{rms}}>\right.$ $1.5 \mathrm{~cm}$ ), however, the equation cannot yield a constant $S_{r}$ for given soil roughness parameter $\left(h_{\mathrm{rms}}, L_{C}\right)$ because the oversimplified assumption of $S_{r}=\sigma_{\mathrm{pp}}^{0} / R_{\mathrm{pp}}$ makes $S_{r}$ vulnerable to the change of $R_{\mathrm{pp}}$. Furthermore, assuming a unique roughness factor in a given $\left(h_{\mathrm{rms}}, L_{C}\right)$ value for $\sigma_{\mathrm{hh}}^{0}$ and $\sigma_{\mathrm{vv}}^{0}$ is not realistic and thus not accurate. Therefore, for better inversion accuracy, more precise simplification for AIEM is required. In this section, we employ a multiplicative model [30] which is capable of modeling radar backscattering for incidence angles up to $60^{\circ}$. We still assume that the radar backscattering is a product of a dielectric factor and a roughness factor while using an undetermined form

$$
\sigma_{\mathrm{hhvv}}^{0}=f_{\mathrm{hhvv}}\left(R_{\mathrm{hh}}\right)^{*} \operatorname{Sr}_{\mathrm{hhvv}}\left(h_{\mathrm{rms}}, L_{C}\right)
$$

where $\sigma_{\mathrm{hhvv}}^{0}=\sigma_{\mathrm{hh}}^{a} \sigma_{\mathrm{vv}}^{b}$. Without a loss of generality, we suppose $|a|+|b|=1$.

By definition, we assume that the dielectric function $f_{\mathrm{hhvv}}\left(R_{\mathrm{hh}}\right)$ only depends on soil dielectric, while the soil roughness function $S r_{\mathrm{hhvv}}$ is exclusively dependent on soil roughness parameters, i.e., $\left(h_{\mathrm{rms}}, L_{C}\right)$ in the scope of this study. After the assumption of (9), its validation step and the formulation follow. The logarithm of (9) form is written

$$
\ln \sigma_{\mathrm{hhvv}}^{0}=\ln f_{\mathrm{hhvv}}\left(R_{\mathrm{hh}}\right)+\ln S r_{\mathrm{hhvv}} .
$$

Obviously, (9) and (10) are the generalized case of (6). An overdetermined linear system is used to derive the values of $\ln f_{\mathrm{hhvv}}\left(R_{\mathrm{hh}}\right)$ and $\ln S r_{\mathrm{hhvv}}$

$$
\left[\begin{array}{l}
\ln \sigma_{\text {hhvv1 }}^{0} \\
\ln \sigma_{\text {hhvv2 }}^{0} \cdots \\
\ln \sigma_{\text {hhvvn*k }}^{0}
\end{array}\right]=\left[\begin{array}{cc}
A_{1} & I \\
A_{2} & I \\
\cdots & \cdots \\
A_{n} & I
\end{array}\right]\left[\begin{array}{l}
\ln f_{\mathrm{hhvv}}\left(R_{\mathrm{hh} 1}\right) \\
\ln f_{\mathrm{hhvv}}\left(R_{\mathrm{hh} 2}\right) \\
\cdots \\
\ln f_{\mathrm{hhvv}}\left(R_{\mathrm{hhn}}\right) \\
\ln S r_{\mathrm{hhvv}}\left(h_{\mathrm{rms} 1}, L_{C 1}\right) \\
\ln S r_{\mathrm{hhvv}}\left(h_{\mathrm{rms} 2}, L_{C 2}\right) \\
\cdots \\
\ln S r_{\mathrm{hhvv}}\left(h_{\mathrm{rmsk}}, L_{C k}\right)
\end{array}\right] .
$$




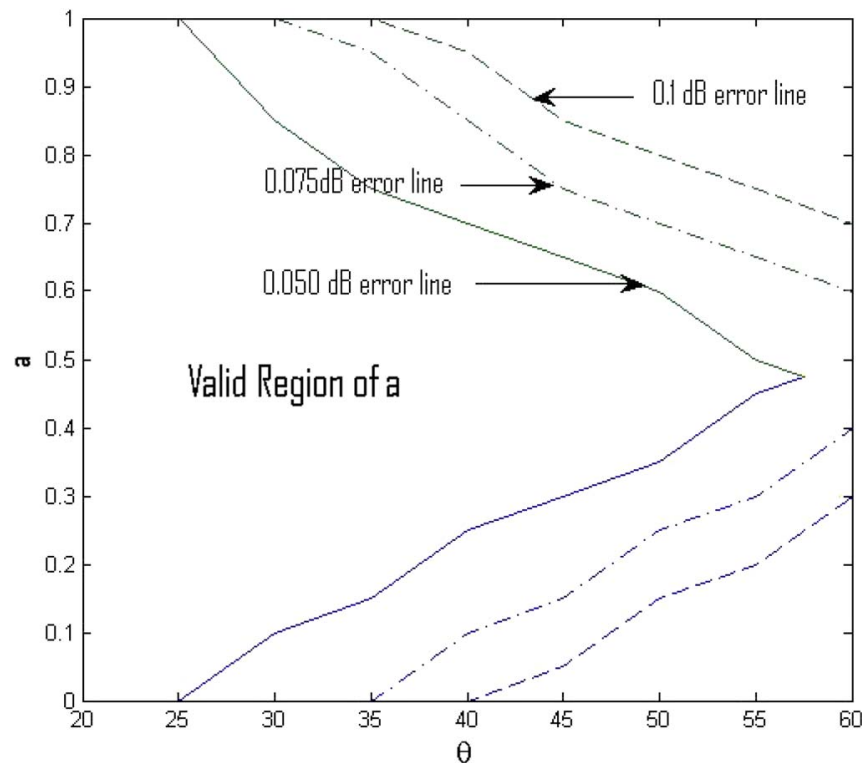

Fig. 2. RMSE contours of (13) for $a$ at different incidence angles. The areas between the two solid/dash dot/dash lines are the valid area in 0.05-, 0.075-, and 0.1-dB rmse, respectively.

The left side of (11) represents AIEM-simulated NRCS at copolarizations calculated from the valid range of the natural surfaces and AIEM input. Supposing that we have $n$ different simulated $R_{\mathrm{hh}}$ values and $k$ different simulated soil roughness $\left(h_{\mathrm{rms}}, L_{C}\right)$ cases, $A_{i}$ stands for a $(k * n)$ matrix with the $i$ th column of one while all the other elements are zero; $I$ stands for a $\left(k^{*} k\right)$ identity matrix. Thus, the unknown vector is an $(n+k)$ dimensional column. There are $(n * k)$ equations in total. By substituting the solution back into the equations, we can validate the assumption made in (9) and (10). If all the equations are well satisfied, (9) and (10) hold. Otherwise, they are invalid. By solving (11) using QR factorization with column pivoting [31], we found that valid $(a, b)$ existed for all mostly used incidence angles $\left(20^{\circ}-60^{\circ}\right)$. Despite that this multiplicative model applies to a wide range of incidence angle, a fixed $R_{\mathrm{hh}}$ at $\theta=20$ can be applied through them because $R_{\mathrm{pp}}\left(\varepsilon_{r}, \theta\right)$ just serves as a transition variable of $\varepsilon$. Then, one can employ a uniform relationship between $R_{\mathrm{hh}}\left(\varepsilon_{r}, 20\right)$ and $\varepsilon_{r}$ once $R_{\mathrm{pp}}$ has been solved. By fitting the solution, we found that simple polynomials were capable of formulating $\ln f\left(R_{\mathrm{hh}}\right)$ and $\ln S r_{\mathrm{hhvv}}\left(h_{\mathrm{rms}}, L_{C}\right)$. Therefore, we can write (10) as

$$
\ln \sigma_{\mathrm{hhvv}}^{0}=\ln f\left(R_{\mathrm{hh}}\right)+\ln S r_{\mathrm{hhvv}}=c o\left(R_{\mathrm{hh}}, h_{\mathrm{rms}}, L_{C}\right)
$$

where

$$
\begin{aligned}
\ln \operatorname{Sr}_{\mathrm{hhvv}}\left(h_{\mathrm{rms}}, L_{C}\right) \\
=a_{\mathrm{sr}}+b_{\mathrm{sr}} \ln h_{\mathrm{rms}}+c_{\mathrm{sr}} \ln L c+d_{\mathrm{sr}}\left(\ln h_{\mathrm{rms}}\right)^{2} \\
\quad+e_{\mathrm{sr}}(\ln L c)^{2}+f_{\mathrm{sr}} \ln h_{\mathrm{rms}} \ln L c+g_{\mathrm{sr}}\left(\ln h_{\mathrm{rms}}\right)^{3} \\
\quad+h_{\mathrm{sr}}(\ln L c)^{3}+i_{\mathrm{sr}} h_{\mathrm{rms}}(\ln L c)^{2}+j_{\mathrm{sr}}\left(\ln h_{\mathrm{rms}}\right)^{2} \ln L c \\
\quad+k_{\mathrm{sr}}\left(\ln h_{\mathrm{rms}}\right)^{4}+l_{\mathrm{sr}}(\ln L c)^{4}+m_{\mathrm{sr}} \ln h_{\mathrm{rms}}(\ln L c)^{3} \\
\quad+n_{\mathrm{sr}}\left(\ln h_{\mathrm{rms}}\right)^{2}(\ln L c)^{2}+o_{\mathrm{sr}}\left(\ln h_{\mathrm{rms}}\right)^{3} \ln L c
\end{aligned}
$$

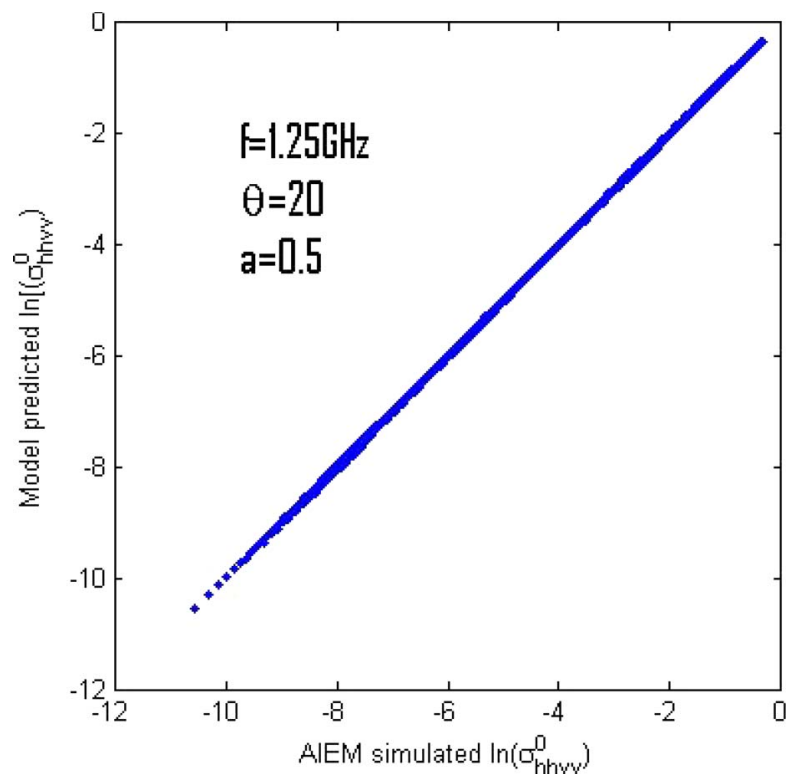

Fig. 3. AIEM simulation versus the right side of (12).

$$
\begin{aligned}
& +p_{\mathrm{sr}} h_{\mathrm{rms}}^{5}+q_{\mathrm{sr}}(\ln L c)^{5}+r_{\mathrm{sr}} h_{\mathrm{rms}}(\ln L c)^{4} \\
& +s_{\mathrm{sr}} h_{\mathrm{rms}}^{2}(\ln L c)^{3}+t_{\mathrm{sr}} h_{\mathrm{rms}}^{3}(\ln L c)^{2} \\
& +u_{\mathrm{sr}} h_{\mathrm{rms}}^{4} \ln L c
\end{aligned}
$$

$\ln f\left(R_{\mathrm{hh}}\right)$

$$
=a_{d}+b_{d} R_{\mathrm{hh}}^{0.5}+c_{d} R_{\mathrm{hh}}+d_{d} R_{\mathrm{hh}}^{1.5}
$$

To obtain the valid region of $a(b=1-a)$, we solve (11) using different $a$ that ranges from zero to one at 0.02 intervals and evaluate the solving standard error. Fig. 2 shows the error counter for $a$ versus $\theta$ plane. For each fixed error value, an up boundary and a low boundary exist in Fig. 2. The region between the two boundaries is the valid region in which the value $a$ enables the solving standard error of (11) smaller than the fixed error value. In Fig. 2, it is observed that, in the same error limit, the smaller the incidence angle, the larger the valid region of $a$ exists. The smallest error occurs when $a$ approaches to 0.5 at all incidence angles. Measured in decibels per power, letting $a=0.5$, the solving standard errors for the incidence angles of $20^{\circ}, 30^{\circ}, 40^{\circ}, 50^{\circ}$, and $60^{\circ}$ are $0.0033 / 1.91 \times 10^{-4}, 0.0068 /$ $2.15 \times 10^{-4}, 0.0173 / 3.84 \times 10^{-4}, 0.0345 / 4.72 \times 10^{-4}$, and $0.0622 / 5.96 \times 10^{-4}$, respectively. Obviously, even in the worst case $\left(\theta=60^{\circ}\right)$, the model error is negligible compared with the noise of radar measurement. The scatter plots of (12) at $20^{\circ}$ incident angle are shown in Fig. 3. Due to the simplification and negligible error of (9) compared with AIEM, it serves as a basis of this study.

\section{Difference (in Decibels) of Double-Incidence Angle Backscattering}

By slightly adjusting $(a, b)$ in the experiment of this paper, setting $(a, b)$ to $(0.5,0.5)$ for small to medium incidence angles and $(0.6,0.4)$ for large incidence angle $\left(>35^{\circ}\right)$, we can minimize the effect of soil moisture in the derived equation 
for double-incidence angle difference (in decibels). Such $(a, b)$ enables the equation $\ln f\left(R_{\mathrm{hh}}\right)=r_{i} \ln \left(R_{\mathrm{hh}}\right)$ to fit very well. We have

$$
\left\{\begin{array}{l}
\ln \sigma_{\mathrm{hhvv}, \theta_{1}}^{0}=r_{1} \ln R_{\mathrm{hh}}+\ln \mathrm{Sr}_{\mathrm{hhvv} 1} \\
\ln \sigma_{\mathrm{hhvv}, \theta_{2}}^{0}=r_{2} \ln R_{\mathrm{hh}}+\ln \mathrm{Sr}_{\mathrm{hhvv} 2} .
\end{array}\right.
$$

By eliminating $R_{\mathrm{hh}}$ from (15), we obtain an equation that is not only simple in form but also relatively uncorrelated to (12), which is

$$
\frac{\ln \sigma_{\mathrm{hhvv}, \theta_{1}}^{0}}{r_{1}}-\frac{\ln \sigma_{\mathrm{hhvv}, \theta_{2}}^{0}}{r_{2}}=\lambda\left(h_{\mathrm{rms}}, L c\right) .
$$

(16) can be precisely fitted using a five-order polynomial

$$
\begin{aligned}
\lambda(s, L c)= & a_{\mathrm{diff}}+b_{\mathrm{diff}} \ln h_{\mathrm{rms}}+c_{\mathrm{diff}} \ln L c+d_{\mathrm{diff}} h_{\mathrm{rms}}^{2} \\
& +e_{\mathrm{diff}}(\ln L c)^{2}+f_{\mathrm{diff}} h_{\mathrm{rms}} \ln L c+g_{\mathrm{diff}} h_{\mathrm{rms}}^{3} \\
& +h_{\mathrm{diff}}(\ln L c)^{3}+i_{\mathrm{diff}} h_{\mathrm{rms}}(\ln L c)^{2} \\
& +j_{\mathrm{diff}} h_{\mathrm{rms}}^{2} \ln L c+k_{\mathrm{diff}}\left(\ln h_{\mathrm{rms}}\right)^{4} \\
& +l_{\mathrm{diff}}(\ln L c)^{4}+m_{\mathrm{diff}} h_{\mathrm{rms}}(\ln L c)^{3} \\
& +n_{\mathrm{diff}} h_{\mathrm{rms}}^{2}(\ln L c)^{2}+o_{\mathrm{diff}} h_{\mathrm{rms}}^{3} \ln L c \\
& +p_{\mathrm{diff}} h_{\mathrm{rms}}^{5}+q_{\mathrm{diff}}(\ln L c)^{5} \\
& +r_{\mathrm{diff}} h_{\mathrm{rms}}(\ln L c)^{4}+s_{\mathrm{diff}} h_{\mathrm{rms}}^{2}(\ln L c)^{3} \\
& +t_{\mathrm{diff}} h_{\mathrm{rms}}^{3}(\ln L c)^{2}+u_{\mathrm{diff}} h_{\mathrm{rms}}^{4} \ln L c .
\end{aligned}
$$

However, it should be noted that measurements of difference in decibels or ratio in power will usually amplify the solving error of (12). In Section II-B, we know that the backscattering of a larger incidence angle introduces a larger error in (12). Thus, if one of the incidence angles is large $\left(>35^{\circ}\right)$, the fitting error of (16) will also be relatively large. In this circumstance, we let the coefficients of one- to three-order roughness terms be functions of $R_{\mathrm{hh}}$, which is written as

$$
\frac{\ln \sigma_{\mathrm{hhvv}, \theta_{1}}^{0}}{r_{1}}-\frac{\ln \sigma_{\mathrm{hhvv}, \theta_{2}}^{0}}{r_{2}}=\lambda\left(R_{\mathrm{hh}}, h_{\mathrm{rms}}, L c\right) .
$$

The complete form of (18) is in (B1) and (B2) of Appendix B with its fitting method described. The scatter plots of 20 versus 30 incidence angles are shown in Fig. 4 in Appendix A.

\section{Coupled Equation for Copolarization Ratio}

Copolarization ratio $\ln \left(\sigma_{\mathrm{hh}}^{0} / \sigma_{\mathrm{vv}}^{0}\right)$ is another measurement we found that has low correlation value to the previous two. When $(a, b)=(0.5,-0.5)$, we can observe from Fig. 5 that the solving error of (12) is unacceptably large. Therefore, the multiplicative model is not capable of including the case of $(a, b)=(0.5,-0.5)$. For small-scale applications, AIEM can be used directly to link copolarized ratio and the ground parameters in

$$
\ln \frac{\sigma_{\mathrm{hh}}^{0}}{\sigma_{\mathrm{vv}}^{0}}=\operatorname{cr}\left(R_{\mathrm{hh}}, h_{\mathrm{rms}}, L_{C}\right) .
$$

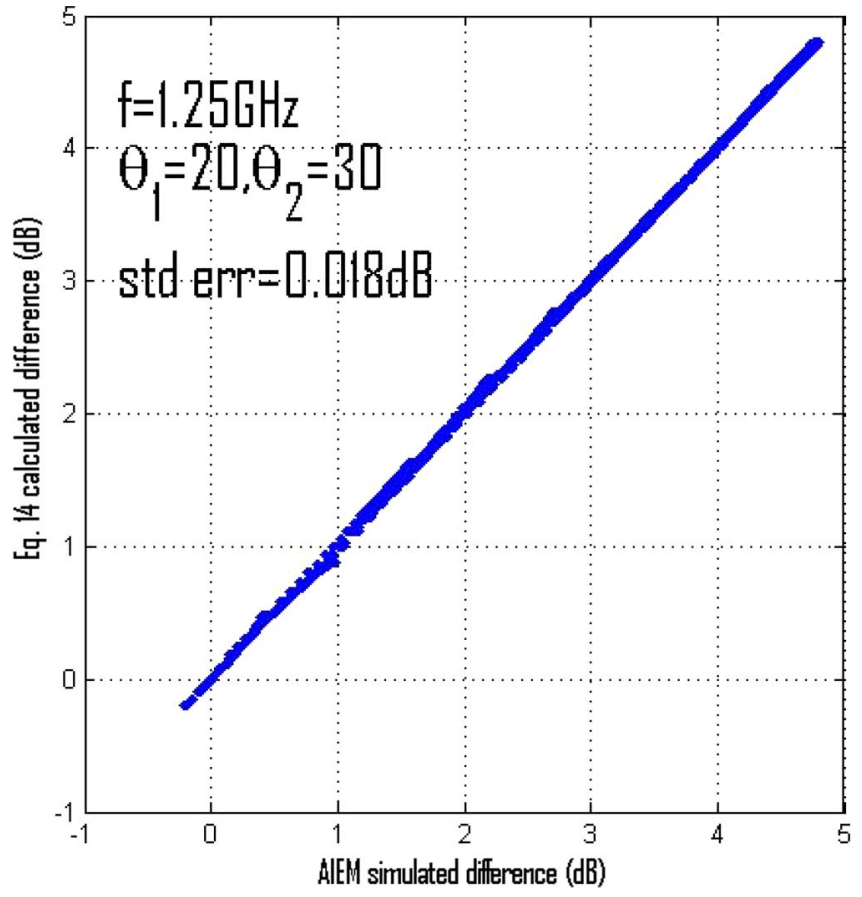

Fig. 4. AIEM simulation versus the right side of (14).

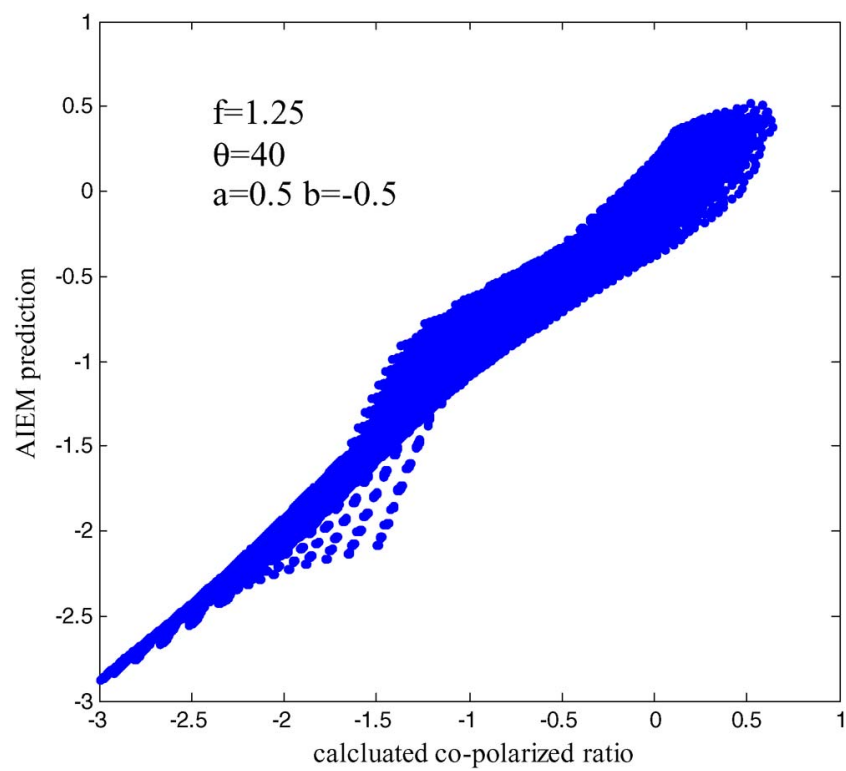

Fig. 5. AIEM simulation versus the right side of (12) when $a=0.5$ and $b=-0.5$.

For large-scale applications, a simpler equation is needed to replace AIEM to decrease the computational expense. In our test, a six-order polynomial which is written in (B3) of Appendix B can fit it precisely.

\section{E. Conversion From to $R_{\mathrm{hh}}$ to $m_{v}$}

After $R_{\mathrm{hh}}$ is solved from the equation set, our final target is to convert it to the volumetric soil moisture. Since the relationship of $R_{\mathrm{hh}}$ and $\varepsilon_{r}$ is straightforward through (7) and $\varepsilon_{r}$ is solely dependent on soil moisture once soil characteristic is known, $m_{v}$ can be immediately obtained by using an up to 2.5 -order 
polynomial that uses the output of (7) to fit the soil moisture in [28] and [29]

$$
\begin{aligned}
m_{v}=a_{m v}+b_{m v} R_{\mathrm{hh}}^{0.5}+c_{m v} R_{\mathrm{hh}} & \\
& +d_{m v} R_{\mathrm{hh}}^{1.5}+e_{m v} R_{\mathrm{hh}}^{2}+f_{m v} R_{\mathrm{hh}}^{2.5}
\end{aligned}
$$

where $a_{m v}-f_{m v}$ depends only on soil characteristics and temperature. The merit of estimating $m_{v}$ from $R_{\mathrm{hh}}$ instead of directly from the equation set is that, for given incidence angle and frequency, all the coefficients in the equation set can remain constant for any soil characteristic and temperature.

\section{INVERTED SOLVING}

\section{A. Nonlinear System}

In Section II, a nonlinear equation set is constructed to solve for the soil moisture without using prior knowledge of soil roughness. The Gaussian-Newtown method is used to solve the equation set since the Jacobian matrix is easy to obtain using the first-order derivative of (12), (16)/(18), and (19). By Taylor formula, we have

$$
\begin{array}{r}
{\left[\begin{array}{c}
c o\left(R_{\mathrm{hh}}^{*}, h_{\mathrm{rms}}^{*}, L_{C}^{*}\right) \\
\lambda\left(R_{\mathrm{hh}}^{*}, h_{\mathrm{rms}}^{*}, L_{C}^{*}\right) \\
c r\left(R_{\mathrm{hh}}^{*}, h_{\mathrm{rms}}^{*}, L_{C}^{*}\right)
\end{array}\right]=\left[\begin{array}{ccc}
\frac{\partial c o}{\partial R_{\mathrm{hh}}} & \frac{\partial c o}{\partial h_{\mathrm{rms}}} & \frac{\partial c o}{\partial L_{C}} \\
\frac{\partial \lambda}{\partial R_{\mathrm{hh}}} & \frac{\partial \lambda}{\partial h_{\mathrm{rms}}} & \frac{\partial \lambda}{\partial L_{C}} \\
\frac{\partial c r}{\partial R_{\mathrm{hh}}} & \frac{\partial c r}{\partial h_{\mathrm{rms}}} & \frac{\partial c r}{\partial L_{C}}
\end{array}\right]\left[\begin{array}{c}
\Delta R_{\mathrm{hh}} \\
\Delta h_{\mathrm{rms}} \\
\Delta L_{C}
\end{array}\right]} \\
+ \\
+\left[\begin{array}{c}
c o\left(R_{\mathrm{hh}}^{\mathrm{ini}}\right) \\
\lambda\left(h_{\mathrm{rms}}^{\mathrm{in}}\right) \\
c r\left(L_{C}^{\mathrm{ini}}\right)
\end{array}\right]
\end{array}
$$

Let

$$
\Delta F^{(i)}=\left[\begin{array}{c}
c o \\
\lambda \\
c r
\end{array}\right]-\left[\begin{array}{c}
c o\left(R_{\mathrm{hh}}^{(i)}, h_{\mathrm{rms}}^{(i)}, L_{C}^{(i)}\right) \\
\lambda\left(R_{\mathrm{hh}}^{(i)}, h_{\mathrm{rms}}^{(i)}, L_{C}^{(i)}\right) \\
c r\left(R_{\mathrm{hh}}^{(i)}, h_{\mathrm{rms}}^{(i)}, L_{C}^{(i)}\right)
\end{array}\right]
$$

where the first term in the right-hand side of (22) stands for the radar measurement.

Let

$$
J=\left[\begin{array}{lll}
\frac{\partial c o}{\partial R_{\mathrm{hh}}} & \frac{\partial c o}{\partial h_{\mathrm{rms}}} & \frac{\partial c o}{\partial L_{C}} \\
\frac{\partial \lambda}{\partial R_{\mathrm{hh}}} & \frac{\partial \lambda}{\partial h_{\mathrm{rms}}} & \frac{\partial \lambda}{\partial L_{C}} \\
\frac{\partial c r}{\partial R_{\mathrm{hh}}} & \frac{\partial c r}{\partial h_{\mathrm{rms}}} & \frac{\partial c r}{\partial L_{C}}
\end{array}\right] .
$$

Using the least squares principle, the iteration formulas are

$$
\begin{gathered}
{\left[\begin{array}{c}
\Delta R_{\mathrm{hh}}^{(i)} \\
\Delta h_{\mathrm{rms}}^{(i)} \\
\Delta L_{C}^{(i)}
\end{array}\right]=\left(J^{T(i)} J^{(i)}\right)^{-1} J^{(i)} \Delta F^{(i)}} \\
{\left[\begin{array}{c}
R_{\mathrm{hh}}^{(i)} \\
h_{\mathrm{rms}}^{(i)} \\
L_{C}^{(i)}
\end{array}\right]=\left[\begin{array}{c}
\Delta R_{\mathrm{hh}}^{(i)} \\
\Delta h_{\mathrm{rms}}^{(i)} \\
\Delta L_{C}^{(i)}
\end{array}\right]+\left[\begin{array}{c}
R_{\mathrm{hh}}^{(i-1)} \\
h_{\mathrm{rms}}^{(i-1)} \\
L_{C}^{(i-1)}
\end{array}\right]}
\end{gathered}
$$

where $i$ stands for the order of the current iteration.

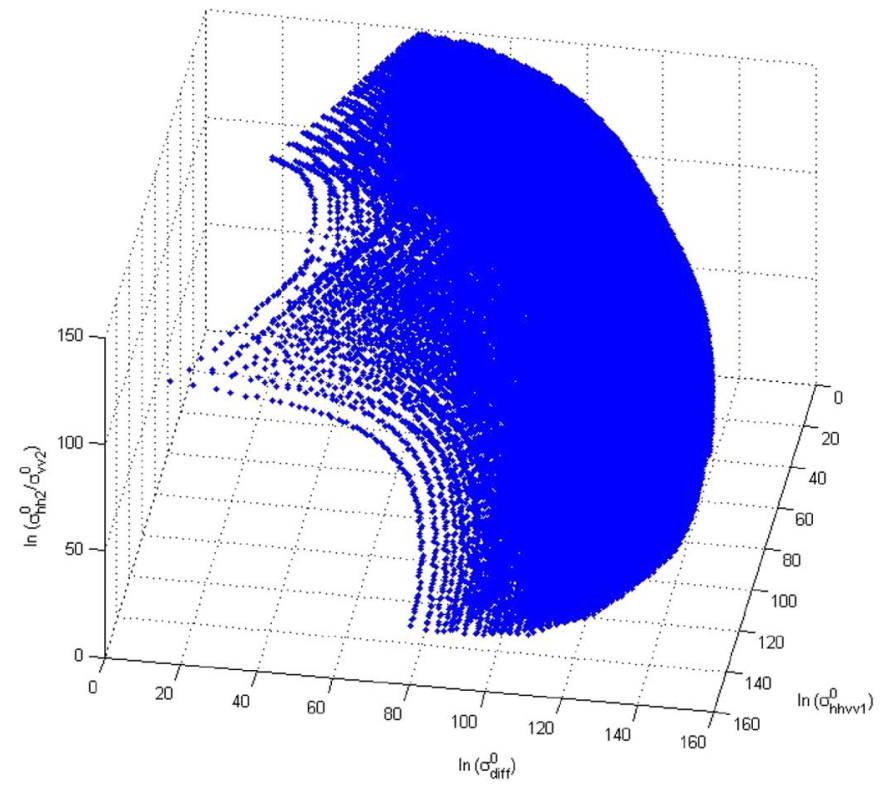

Fig. 6. Illustration of 3D-LUT cloud.

Note that if AIEM is directly used in (19), its derivative of (19) must be approximated by its difference.

\section{B. Establishment and Use of LUT}

To circumvent local optimum solutions through iterations, a good initial guess is required. This study uses a 3D-LUT method to obtain the initial guess for any given combination of measurements, i.e.,

$$
\begin{gathered}
\left(\ln \sigma_{\mathrm{hhvv}, \text { inc } 1}^{0}, \ln \frac{\sigma_{\mathrm{hh}, \text { inc2 }}^{0}}{\sigma_{\mathrm{vv}, \text { inc2 } 2}^{0}}, \frac{\ln \sigma_{\mathrm{hhvv}, \text { inc1 }}^{0}}{r_{1}}-\frac{\ln \sigma_{\mathrm{hhvv}, \text { inc2 }}^{0}}{r_{2}}\right) \\
\sim\left(R_{\mathrm{hh} \mid \text { ini }}, h_{\mathrm{rms} \mid \text { ini }}, L_{C \mid \text { ini }}\right) .
\end{gathered}
$$

Dense AIEM simulation data are used to fill the 3D-LUT. When the measurements fall between the table grids, the nearest neighbor method is used to find the initial guess. Fig. 6 is used to show the 3D-LUT. In Fig. 6, three axes represent the three simulated radar measurements. For each table, the value at a given point $(x, y, z)$ stands for one unknown; it is $R_{\mathrm{hh} \mid \mathrm{ini}}$, $h_{\text {rmslini }}$, or $L_{C \mid \text { ini }}$ in this study. Thus, we need three 3D-LUTs in total. To reduce the computation, we use the uniform table grids which are adequate to give the initial guess.

\section{DATA AND VALIDATION}

The validation work of this study consists of two parts: 1) validation by simulated solving and 2) validation by field data. Simulated solving validation uses simulated radar backscattering data as observations and preset soil parameters as ground truth. Simulated validation is indeed significant because it verifies whether the proposed method can work throughout the whole parameter range, which is often not available using field data. More importantly, simulated solving validation can be repeated and compared by readers. To thoroughly 


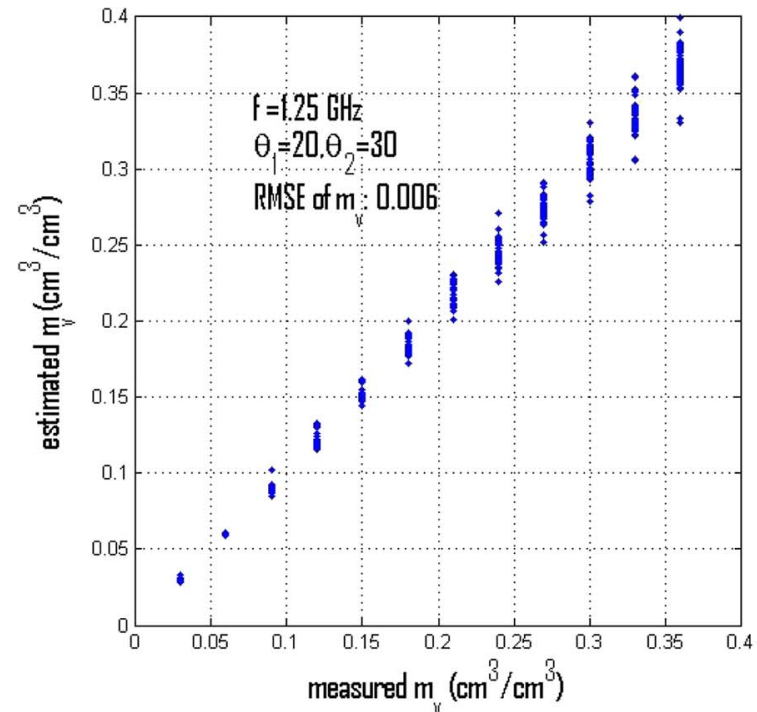

(a)

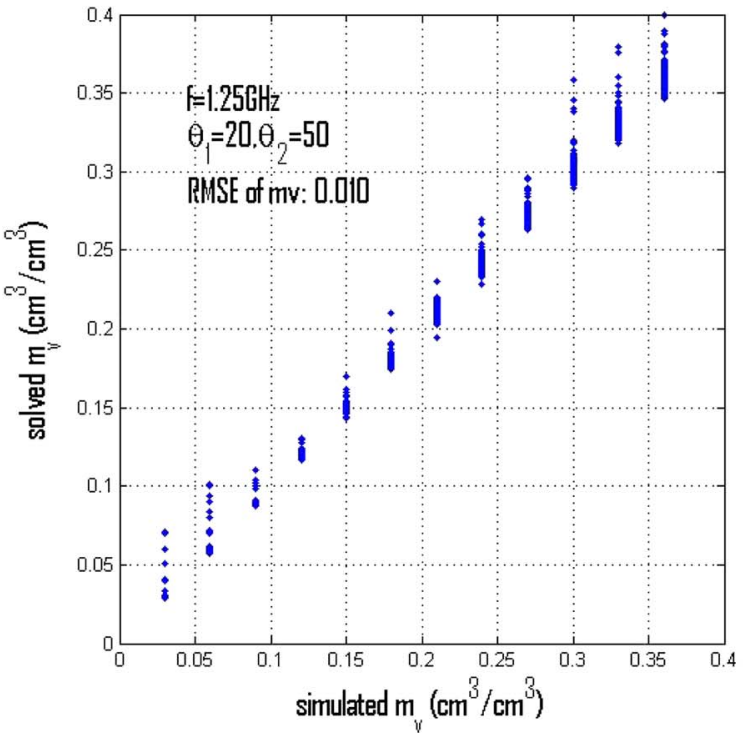

(c)

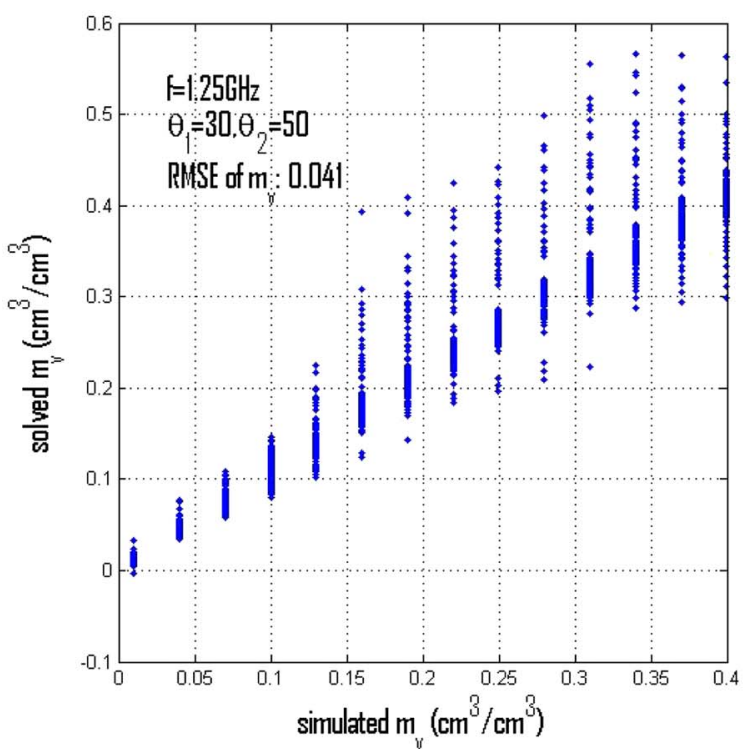

(e)

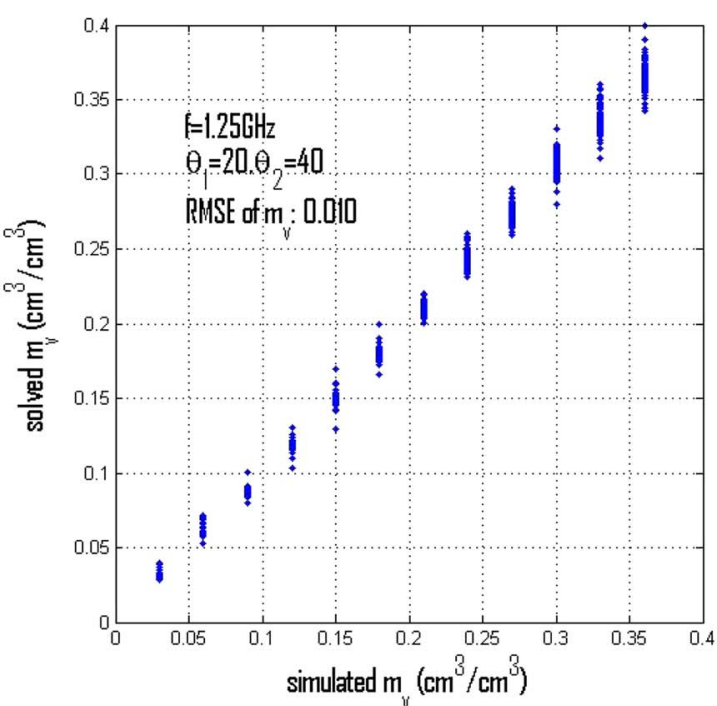

(b)

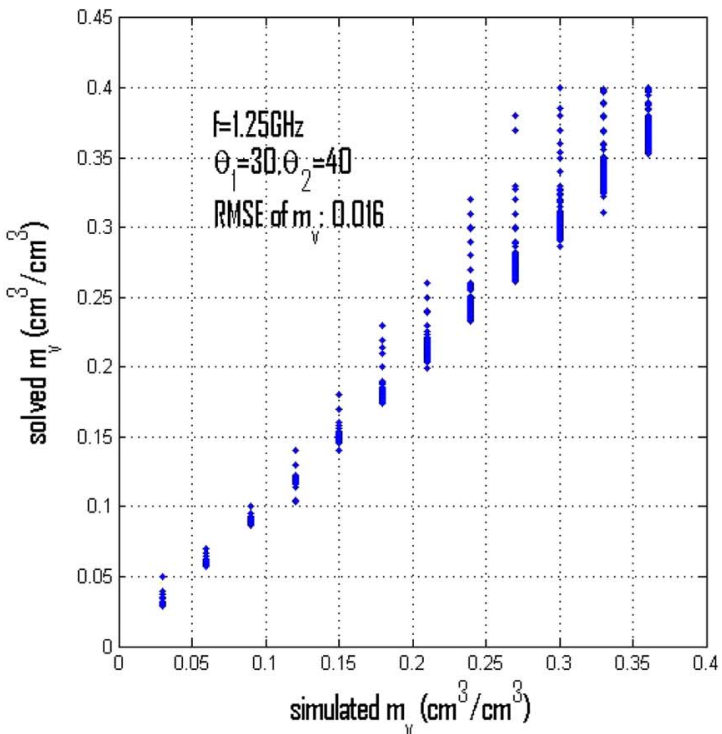

(d)

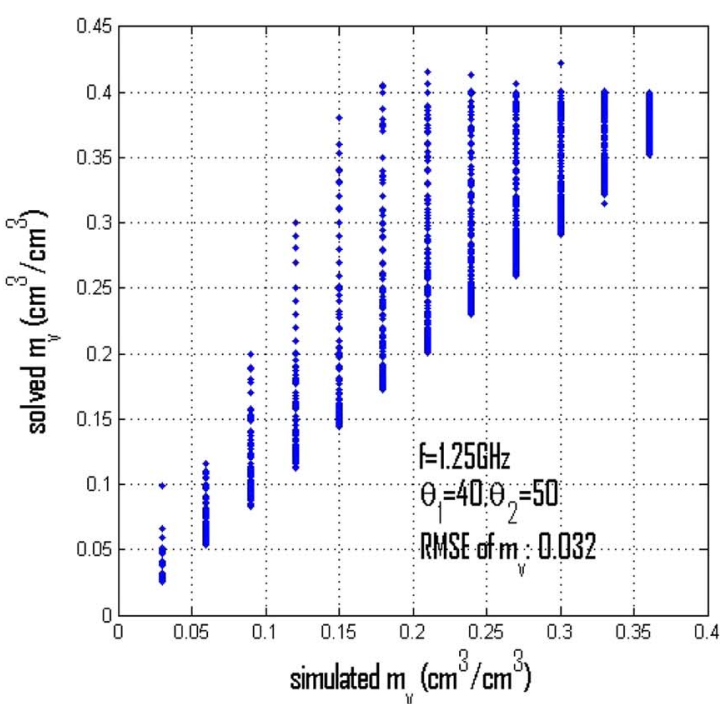

(f)

Fig. 7. Scatter plots and rmse of solved $m_{v}$ versus simulated $m_{v}$. 


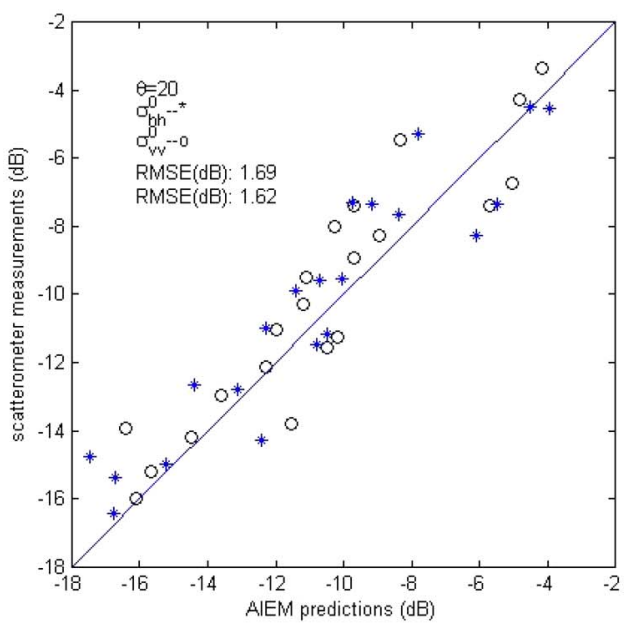

(a)

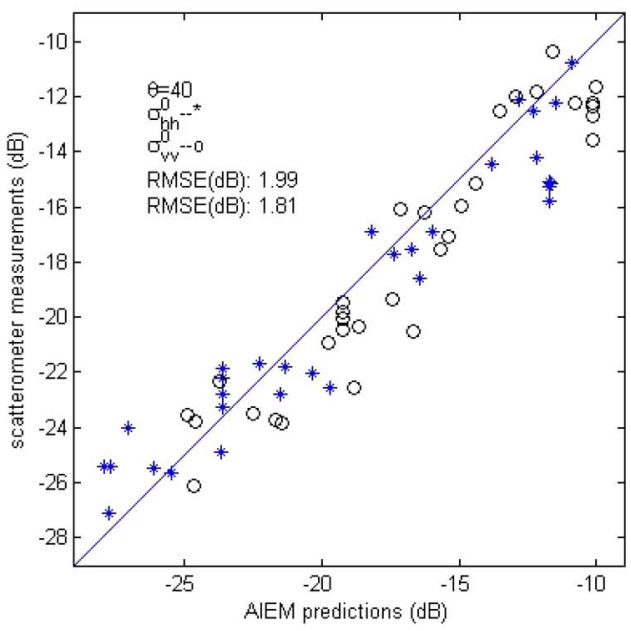

(c)

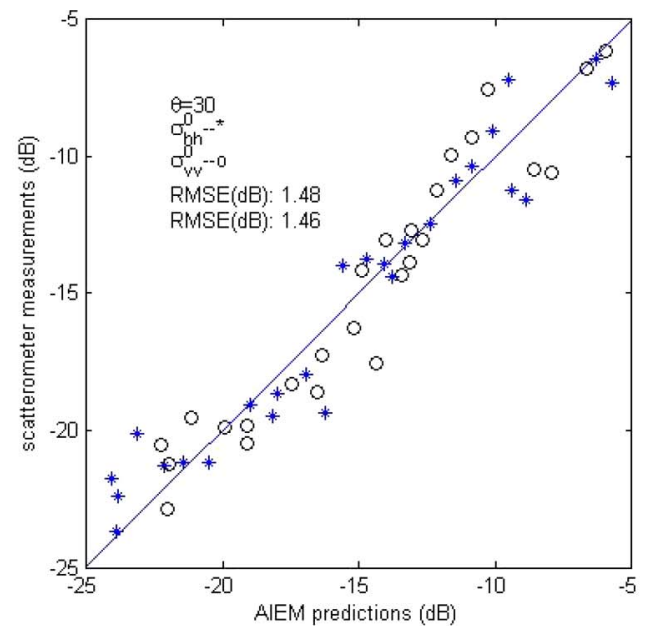

(b)

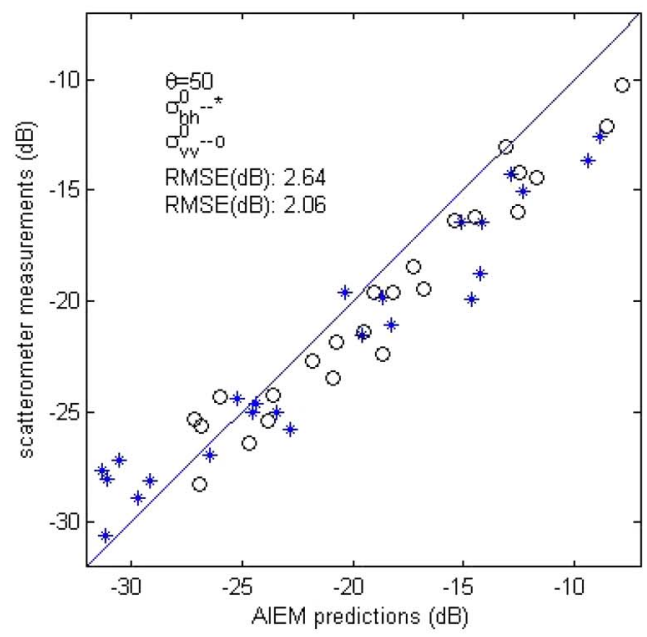

(d)

Fig. 8. Validation of AIEM by the experimental data.

validate the effectiveness of the proposed model, we performed the validation on six different combinations of the doubleincidence angles, which are $20^{\circ}$ versus $30^{\circ}, 20^{\circ}$ versus $40^{\circ}$, $20^{\circ}$ versus $50^{\circ}, 30^{\circ}$ versus $40^{\circ}, 30^{\circ}$ versus $50^{\circ}$, and $40^{\circ}$ versus $50^{\circ}$. These groups correspond to the configurations of our field data. Fig. 7 shows the root-mean-square error (rmse) and scatter plots of simulated soil moisture versus the solved ones.

For field data validation, the University of Michigan polscatterometer experimental data in 1991 [20] are used in this study. In this data set, the intensities of $\mathrm{HH} / \mathrm{VV} / 0.5(\mathrm{HV}+$ $\mathrm{VH})$ at $20^{\circ}-60^{\circ}$ incidence angle that covered four bare soil sites of Ypsilanti in 1991 are available; $h_{\mathrm{rms}}$ varies from 0.55 to $3.47 \mathrm{~cm}, L_{C}$ varies from 6.9 to $11 \mathrm{~cm}$, and $m_{v}$ ranges from $0.7 \%$ to $20 \% \mathrm{~cm}^{3} / \mathrm{cm}^{3}$. Thus, the roughness parameters span roughly the whole valid region of AIEM, and the dynamic range of soil moisture is adequate. It is important to validate the accuracy of AIEM to predict the bare surface backscattering before use. Due to the adoption of only the copolarized backscattering, the single scattering version of AIEM is adequate in the scope of this study, while calculating multiple scattering is time consuming and omitted. The measured soil parameters $\left(\varepsilon_{r}, h_{\mathrm{rms}}\right.$ and $\left.L_{C}\right)$ in our field data are input into
AIEM; then, the output predictions of both polarizations are compared to scatterometer measurements in Fig. 8. In Fig. 8, the rmse values of copolarizations between AIEM prediction and radar backscattering are $1.69 / 1.62 \mathrm{~dB}$ at $20^{\circ}, 1.46 / 1.48 \mathrm{~dB}$ at $30^{\circ}, 1.96 / 1.75 \mathrm{~dB}$ at $40^{\circ}$, and $2.2 / 2.1 \mathrm{~dB}$ at $50^{\circ}$, respectively. It is observed that AIEM is sufficiently accurate, particularly at small and medium incidence angles. At $30^{\circ}$, AIEM achieves the best accuracy. More details of these data are available in [20]. The rmse and scatter plots of estimated $m_{v}$ versus in situ measurements are shown in Fig. 9.

In Fig. 9, two facts are observed. First, the inversion error of soil moisture brought by the numerical solving is usually acceptable in the presence of a low incidence angle, which is $20^{\circ}$ in this experiment, while the error grows with the increase of the smaller incidence angle and becomes unacceptable when both incidence angles become large, which are $40^{\circ}$ and $50^{\circ}$ in this experiment. In conjunction, Figs. 7 and 9 show that, for both high incidence angles, the three employed measurements may become more correlated and make the equations less independent. Second, the error tends to grow a little larger for wet surfaces. The multiplicative nature that the dielectric factor exhibits in the total backscattering power and the multiplicative noiselike radar signal might contribute to this. Another reason 


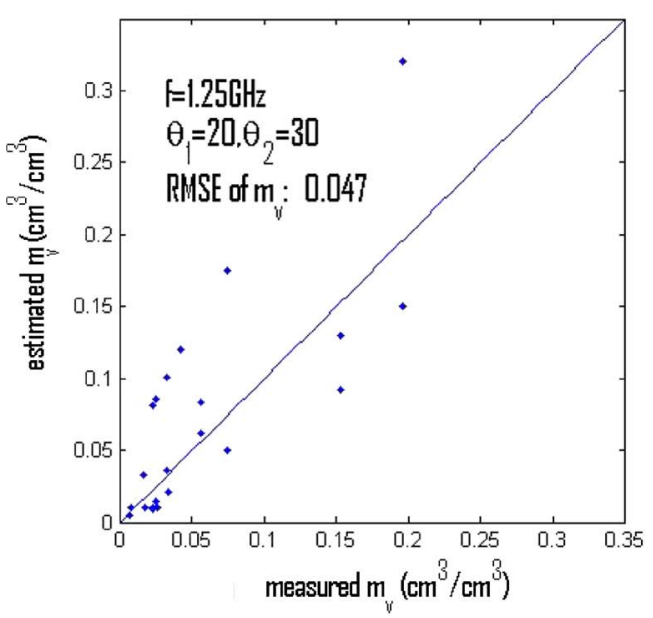

(a)

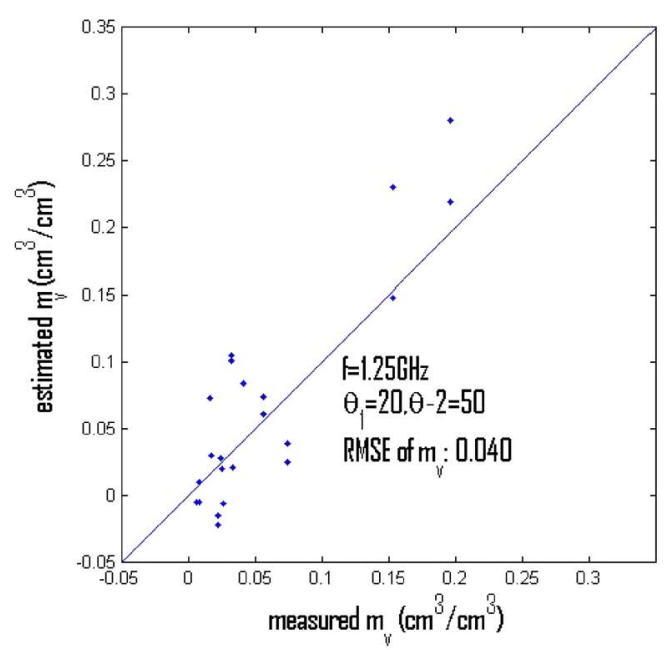

(c)

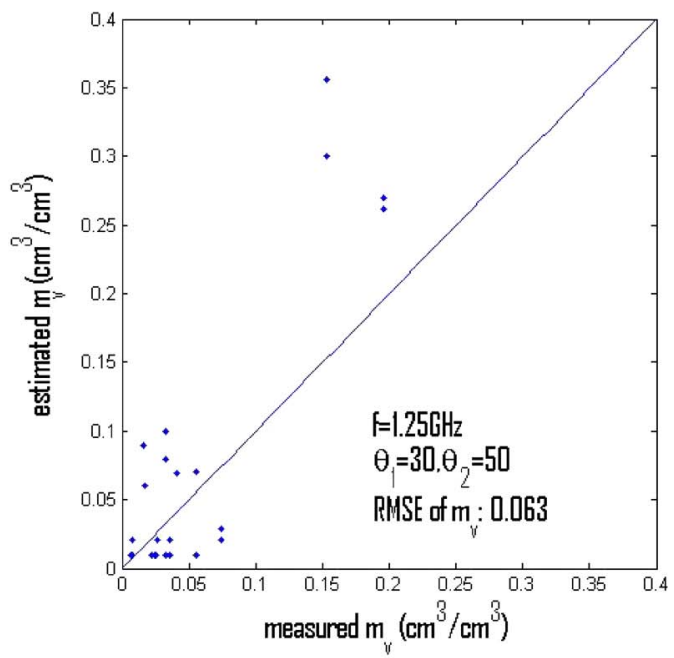

(e)

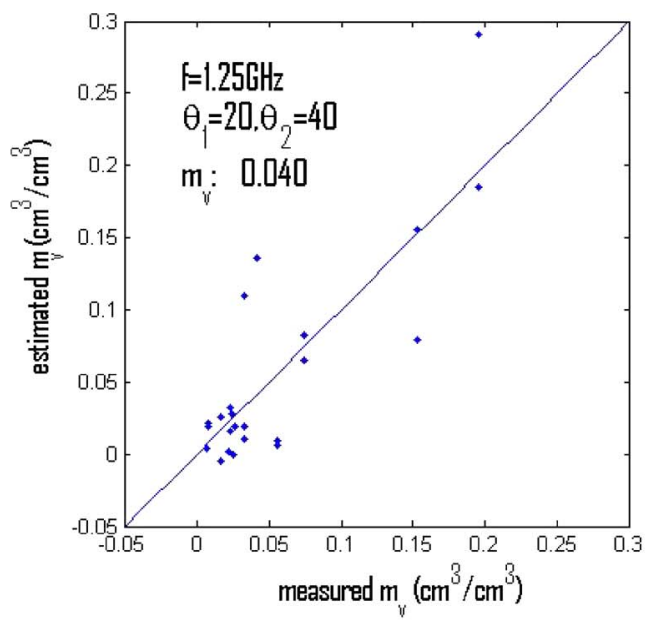

(b)

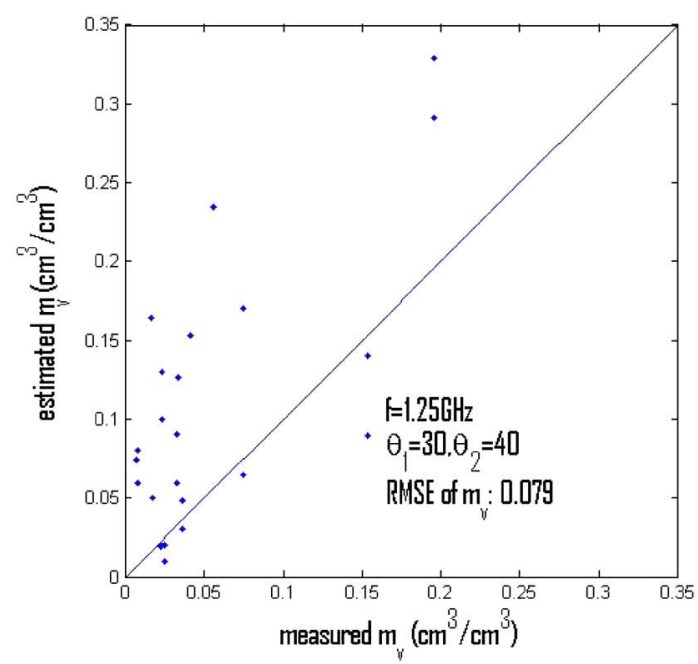

(d)

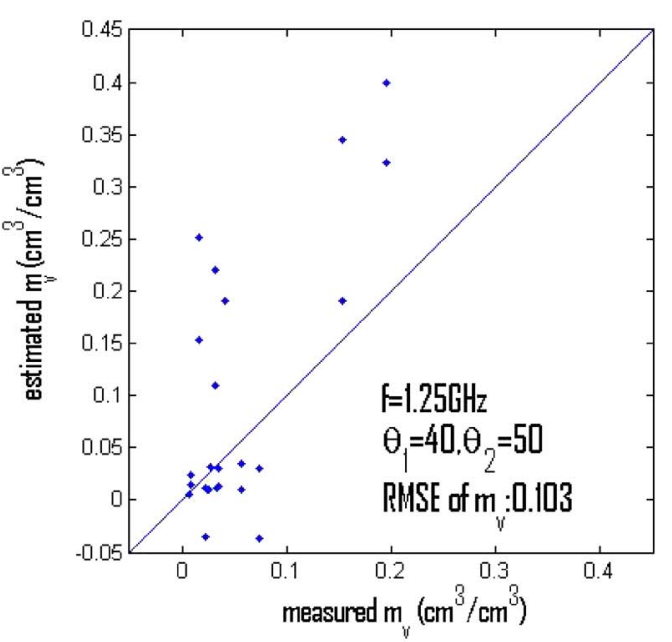

(f)

Fig. 9. Scatter plots and rmse of model inverted $m_{v}$ versus the in situ measurements at different combinations of incidence angles.

is that the radar backscattering is less sensitive to the high soil moisture content than to the low.

In summary, for the application of bare surface soil moisture mapping using the proposed double-incidence angle model at
L-band, it is recommended to include at least one low or medium incidence angle in the pair angles. This attribute coincides with the low incidence angle preference for soil moisture mapping over vegetated areas. 


\section{APPENDIX A}

Forward validation scatter plots of the proposed equations are listed in Appendix A.

\section{APPENDIX B}

$$
\begin{aligned}
\lambda\left(R_{\mathrm{hh}}, s, L c\right)= & a_{\mathrm{diff}}\left(R_{\mathrm{hh}}\right)+b_{\mathrm{diff}}\left(R_{\mathrm{hh}}\right) \ln h_{\mathrm{rms}} \\
& +c_{\mathrm{diff} 3}\left(R_{\mathrm{hh}}\right) \ln L c \\
& +d_{\mathrm{diff}}\left(R_{\mathrm{hh}}\right) h_{\mathrm{rms}}^{2}+e_{\mathrm{diff}}\left(R_{\mathrm{hh}}\right)(\ln L c)^{2} \\
& +f_{\mathrm{diff}}\left(R_{\mathrm{hh}}\right) h_{\mathrm{rms}} \ln L c \\
& +g_{\mathrm{diff}}\left(R_{\mathrm{hh}}\right) h_{\mathrm{rms}}^{3}+h_{\mathrm{diff}}\left(R_{\mathrm{hh}}\right)(\ln L c)^{3} \\
& +i_{\mathrm{diff}}\left(R_{\mathrm{hh}}\right) h_{\mathrm{rms}}(\ln L c)^{2} \\
& +j_{\mathrm{diff}}\left(R_{\mathrm{hh}}\right) h_{\mathrm{rms}}^{2} \ln L c \\
& +k_{\mathrm{diff}}\left(\ln h_{\mathrm{rms}}\right)^{4}+l_{\mathrm{diff}}(\ln L c)^{4} \\
& +m_{\mathrm{diff}} h_{\mathrm{rms}}(\ln L c)^{3} \\
& +n_{\mathrm{diff}} h_{\mathrm{rms}}^{2}(\ln L c)^{2}+o_{\mathrm{diff}} h_{\mathrm{rms}}^{3} \ln L c \\
& +p_{\mathrm{diff}} h_{\mathrm{rms}}^{5} \\
& +q_{\mathrm{diff}}(\ln L c)^{5}+r_{\mathrm{diff}} h_{\mathrm{rms}}(\ln L c)^{4} \\
& +s_{\mathrm{diff}} h_{\mathrm{rms}}^{2}(\ln L c)^{3}+t_{\mathrm{diff}} h_{\mathrm{rms}}^{3}(\ln L c)^{2} \\
& +u_{\mathrm{diff}} h_{\mathrm{rms}}^{4} \ln L c .
\end{aligned}
$$

In (B1), the coefficients of low-order (1-3) roughness terms are functions of $R_{\mathrm{hh}}$. The dielectric factor and roughness factor are partly coupled in (B1). In (B1), the coefficients of the high-order roughness coefficients $k_{\text {diff }} \sim u_{\text {diff }}$ are determined by (B2). After subtracting these high orders, the dielectric dependent coefficients $a_{\text {diff }}\left(R_{\mathrm{hh}}\right) \sim j_{\text {diff }}\left(R_{\mathrm{hh}}\right)$ can be refitted by using a loop fitting with respect of $R_{\mathrm{hh}}$. In each iteration, $a_{\text {diff }}\left(R_{\mathrm{hh}}\right) \sim j_{\text {diff }}\left(R_{\mathrm{hh}}\right)$ in (B4) is refitted using a fixed $R_{\mathrm{hh}}$. Then, we fit (B2) using these values

$$
\begin{aligned}
a_{\mathrm{diff}}( & \left.R_{\mathrm{hh}}\right) \sim j_{\mathrm{diff}}\left(R_{\mathrm{hh}}\right) \\
= & \frac{a+c R_{\mathrm{hh}}^{0.5}+e R_{\mathrm{hh}}+g R_{\mathrm{hh}}^{1.5}+i R_{\mathrm{hh}}^{2}+k R_{\mathrm{hh}}^{2.5}}{1+b R_{\mathrm{hh}}^{0.5}+d R_{\mathrm{hh}}+f R_{\mathrm{hh}}^{1.5}+h R_{\mathrm{hh}}^{2}+j R_{\mathrm{hh}}^{2.5}} .
\end{aligned}
$$

For large-scale applications, using (B3) instead of AIEM in (16) will reduce the computational expense significantly with an even better solving accuracy because the derivative is analytical

$$
\begin{aligned}
\ln \frac{\sigma_{\mathrm{hh}}^{0}}{\sigma_{\mathrm{vv}}^{0}}= & c r\left(R_{\mathrm{hh}}, h_{\mathrm{rms}}, L_{C}\right) \\
= & f_{1}\left(R_{\mathrm{hh}}\right)+f_{2}\left(R_{\mathrm{hh}}\right) \ln h_{\mathrm{rms}}+f_{3}\left(R_{\mathrm{hh}}\right) \ln L c \\
& +f_{4}\left(R_{\mathrm{hh}}\right) h_{\mathrm{rms}}^{2}+f_{5}\left(R_{\mathrm{hh}}\right)(\ln L c)^{2} \\
& +f_{6}\left(R_{\mathrm{hh}}\right) h_{\mathrm{rms}} \ln L c+f_{7}\left(R_{\mathrm{hh}}\right) h_{\mathrm{rms}}^{3} \\
& +f_{8}\left(R_{\mathrm{hh}}\right)(\ln L c)^{3}+f_{9}\left(R_{\mathrm{hh}}\right) h_{\mathrm{rms}}(\ln L c)^{2} \\
& +f_{10}\left(R_{\mathrm{hh}}\right) h_{\mathrm{rms}}^{2} \ln L c+f_{11}\left(R_{\mathrm{hh}}\right)\left(\ln h_{\mathrm{rms}}\right)^{4} \\
& +f_{12}\left(R_{\mathrm{hh}}\right)(\ln L c)^{4}+f_{13}\left(R_{\mathrm{hh}}\right) h_{\mathrm{rms}}(\ln L c)^{3}
\end{aligned}
$$

$$
\begin{aligned}
& +f_{14}\left(R_{\mathrm{hh}}\right) h_{\mathrm{rms}}^{2}(\ln L c)^{2}+f_{15}\left(R_{\mathrm{hh}}\right) h_{\mathrm{rms}}^{3} \ln L c \\
& +f_{16}\left(R_{\mathrm{hh}}\right) h_{\mathrm{rms}}^{5}+f_{17}\left(R_{\mathrm{hh}}\right)(\ln L c)^{5} \\
& +f_{18}\left(R_{\mathrm{hh}}\right) h_{\mathrm{rms}}(\ln L c)^{4}+f_{19}\left(R_{\mathrm{hh}}\right) h_{\mathrm{rms}}^{2}(\ln L c)^{3} \\
& +f_{20}\left(R_{\mathrm{hh}}\right) h_{\mathrm{rms}}^{3}(\ln L c)^{2}+f_{21}\left(R_{\mathrm{hh}}\right) h_{\mathrm{rms}}^{4} \ln L c \\
& +f_{22}\left(R_{\mathrm{hh}}\right) h_{\mathrm{rms}}^{6}+f_{23}\left(R_{\mathrm{hh}}\right)(\ln L c)^{6} .
\end{aligned}
$$

The values of $f_{i}\left(R_{\mathrm{hh}}\right)$ in (B3) can be obtained by solving a different overdetermined system than (11)

$$
\begin{aligned}
{\left[\begin{array}{l}
c r\left(R_{\mathrm{hh} 1}, h_{\mathrm{rms} 1}, L c_{1}\right) \\
\operatorname{cr}\left(R_{\mathrm{hh} 1}, h_{\mathrm{rms} 2}, L c_{2}\right) \\
\ldots \\
c r\left(R_{\mathrm{hh} 1}, h_{\mathrm{rmsk}}, L c_{k}\right) \\
c r\left(R_{\mathrm{hh} 2}, h_{\mathrm{rms} 1}, L c_{1}\right) \\
\cdots \\
c r\left(R_{\mathrm{hhn}}, h_{\mathrm{rmsk}}, L c_{k}\right)
\end{array}\right] } \\
=\left[\begin{array}{lll}
A_{C} & \\
& A_{C} & \\
& \\
&
\end{array}\right]\left[\begin{array}{l}
f_{1}\left(R_{\mathrm{hh} 1}\right) \\
f_{2}\left(R_{\mathrm{hh} 1}\right) \\
\cdots \\
f_{23}\left(R_{\mathrm{hh} 1}\right) \\
\cdots \\
f_{1}\left(R_{\mathrm{hhn}}\right) \\
f_{2}\left(R_{\mathrm{hhn}}\right) \\
\cdots \\
f_{23}\left(R_{\mathrm{hhn}}\right)
\end{array}\right]
\end{aligned}
$$

where $\mathrm{cr}$ means the logarithm of simulated copolarization ratio and

$$
A_{C}=\left[\begin{array}{cccc}
t s_{1}^{1} & t s_{2}^{1} & \cdots & t s_{23}^{1} \\
t s_{1}^{2} & t s_{2}^{2} & \cdots & t s_{23}^{2} \\
\cdots & \cdots & \cdots & \cdots \\
t s_{1}^{k} & t s_{2}^{k} & \cdots & t s_{23}^{k}
\end{array}\right]
$$

where $t s_{i}^{j}$ stands for the $j$ th roughness term in (B3) using the $i$ th $\left(h_{\mathrm{rms}}, L_{C}\right)$ value of the total $k$ different roughness combinations. Then, we fit the $f_{i}\left(R_{\mathrm{hh}}\right)$ values also using (B2).

\section{ACKNOWLEDGMENT}

Author X. Shen would like to thank the Principal Investigators in Washita 1992 for the pol-scatterometer experimental data used in this paper. He would also like to thank Dr. M. M. Rahman for his kind explanation for his paper via e-mail which provided the major inspiration of this work.

\section{REFERENCES}

[1] A. L. Cognard, C. Loumagne, M. Normand, P. Olivier, C. Ottlé, D. VidalMadjar, S. Louahala, and A. Vidal, "Evaluation of the ERS 1/synthetic aperture radar capacity to estimate surface soil moisture: Two-year results over the Naizin watershed," Water Resources Res., vol. 31, no. 4, pp. 975982, 1995.

[2] H. C. Macdonald and W. P. Waite, "Soil moisture detection with imaging radars," Water Resources Res., vol. 7, no. 1, pp. 100-110, 1971.

[3] A. K. Fung, Microwave Scattering and Emission Models and Their Applications. Boston, MA: Artech House, 1994.

[4] H. Mametsa, F. Koudogbo, and P. Combes, "Application of IEM and radiative transfer formulations for bistatic scattering of rough surfaces," in Proc. IGARSS, 2002, pp. 662-664. 
[5] F. T. Ulaby, R. K. Moore, and A. K. Fung, Microwave Remote Sensing: Active and Passive. London, U.K.: Artech House, 1986, pp. 926-966.

[6] L. Tsang, J. A. Kong, and K. H. Ding, Scattering of Electromagnetic Waves. New York: Wiley, 2000

[7] J. L. Alvarez-Perez, "An extension of the IEM/IEMM surface scattering model," Waves Random Complex Media, vol. 11, no. 3, pp. 307-329, 2001.

[8] T. D. Wu, K. Chen, J. Shi, and A. K. Fung, "A transition model for the reflection coefficient in surface scattering," IEEE Trans. Geosci. Remote Sens., vol. 39, no. 9, pp. 2040-2050, Sep. 2002.

[9] K. Chen, T. D. Wu, L. Tsang, Q. Li, J. Shi, and A. K. Fung, "Emission of rough surfaces calculated by the integral equation method with comparison to three-dimensional moment method simulations," IEEE Trans. Geosci. Remote Sens., vol. 41, no. 1, pp. 90-101, Jan. 2003.

[10] T. D. Wu, K. S. Chen, J. Shi, H.-W. Lee, and A. K. Fung, "A study of an AIEM model for bistatic scattering from randomly rough surfaces," IEEE Trans. Geosci. Remote Sens., vol. 46, no. 9, pp. 2584-2598, Sep. 2008.

[11] M. Piles, D. Entekhabi, and A. Camps, "A change detection algorithm for retrieving high-resolution soil moisture from SMAP radar and radiometer observations," IEEE Trans. Geosci. Remote Sens., vol. 47, no. 12, pp. 4125-4131, Dec. 2009.

[12] S. B. Kim, L. Tsang, J. T. Johnson, S. Huang, J. J. van Zyl, and E. G. Njoku, "Soil moisture retrieval using time-series radar observations over bare surfaces," IEEE Trans. Geosci. Remote Sens., vol. 50, no. 5, pp. 1853-1863, May 2012.

[13] Y. Kim and J. J. van Zyl, "A time-series approach to estimate soil moisture using polarimetric radar data," IEEE Trans. Geosci. Remote Sens., vol. 47, no. 8, pp. 2519-2527, Aug. 2009.

[14] E. Altese, O. Bolognani, M. Mancini, and P. A. Troch, "Retrieving soil moisture over bare soil from ERS 1 synthetic aperture radar data: Sensitivity analysis based on a theoretical surface scattering model and field data," Water Resources Res., vol. 32, no. 3, pp. 653-661, Mar. 1996.

[15] G. F. Biftu and T. Y. Gan, "Retrieving near-surface soil moisture from Radarsat SAR data," Water Resources Res., vol. 35, no. 5, pp. 1569-1579, May 1999.

[16] P. C. Dubois, J. Van Zyl, and T. Engman, "Measuring soil moisture with imaging radars," IEEE Trans. Geosci. Remote Sens., vol. 33, no. 4, pp. 915-926, Jul. 1995.

[17] K. Song, X. Zhou, and Y. Fan, "Empirically adopted IEM for retrieval of soil moisture from radar backscattering coefficients," IEEE Trans. Geosci. Remote Sens., vol. 47, no. 6, pp. 1662-1672, Jun. 2009.

[18] N. Verhoest, B. De Baets, F. Mattia, G. Satalino, C. Lucau, and P. Defourny, "A possibilistic approach to soil moisture retrieval from ERS synthetic aperture radar backscattering under soil roughness uncertainty," Water Resources Res., vol. 43, no. 7, p. W07 435, Jul. 2007.

[19] R. Bindlish and A. P. Barros, "Multifrequency soil moisture inversion from SAR measurements with the use of IEM," Remote Sens. Environ., vol. 71, no. 1, pp. 67-88, Jan. 2000

[20] Y. Oh, "Quantitative retrieval of soil moisture content and surface roughness from multipolarized radar observations of bare soil surfaces," IEEE Trans. Geosci. Remote Sens., vol. 42, no. 3, pp. 596-601, Mar. 2004.

[21] Y. Oh, K. Sarabandi, and F. T. Ulaby, "Semi-empirical model of the ensemble-averaged differential Mueller matrix for microwave backscattering from bare soil surfaces," IEEE Trans. Geosci. Remote Sens., vol. 40, no. 6, pp. 1348-1355, Jun. 1992.

[22] M. Rahman, M. Moran, D. Thoma, R. Bryant, C. D. H. Collins, T. Jackson, B. J. Orr, and M. Tischler, "Mapping surface roughness and soil moisture using multi-angle radar imagery without ancillary data," Remote Sens. Environ., vol. 112, no. 2, pp. 391-402, Feb. 2008.

[23] J. Shi, J. Wang, A. Y. Hsu, P. E. O’Neill, and E. T. Engman, "Estimation of bare surface soil moisture and surface roughness parameter using L-band SAR image data," IEEE Trans. Geosci. Remote Sens., vol. 35, no. 5, pp. 1254-1266, Sep. 1997.

[24] M. Zribi and M. Dechambre, "A new empirical model to retrieve soil moisture and roughness from C-band radar data," Remote Sens. Environ., vol. 84, no. 1, pp. 42-52, Jan. 2003

[25] M. Zribi, N. Baghdadi, and C. Guerin, "Analysis of surface roughness heterogeneity and scattering behavior for radar measurements," IEEE Trans. Geosci. Remote Sens., vol. 44, no. 9, pp. 2438-2444, Sep. 2006.

[26] M. Owe and A. A. Van de Griend, "Comparison of soil moisture penetration depths for several bare soils at two microwave frequencies and implications for remote sensing," Water Resources Res., vol. 34, no. 9, pp. 2319-2327, Sep. 1998.

[27] J. S. Lee and E. Pottier, Polarimetric Radar Imaging: From Basics to Applications. Boca Raton, FL: CRC Press.

[28] M. C. Dobson, F. T. Ulaby, M. T. Hallikainen, and M. A. El-Rayes, "Microwave dielectric behavior of wet soil-Part II: Dielectric mixing models," IEEE Trans. Geosci. Remote Sens., vol. GE-23, no. 1, pp. 3546, Jan. 1985.

[29] N. R. Peplinski, F. T. Ulaby, and M. C. Dobson, "Dielectric properties of soils in the 0.3-1.3-GHz range," IEEE Trans. Geosci. Remote Sens., vol. 33, no. 3, pp. 803-807, May 1995.

[30] X. Shen, Y. Hong, Q. Qin, W. Yuan, S. Chen, S. Zhao, and T. Grout, "Orientation angle calibration for bare soil moisture estimation using fully polarimetric SAR data," IEEE Trans. Geosci. Remote Sens., vol. 49, no. 12, pp. 4987-4996, Dec. 2011

[31] L. N. Trefethen and D. Bau, Numerical Linear Algebra. Philadelphia, PA: SIAM, 1997.

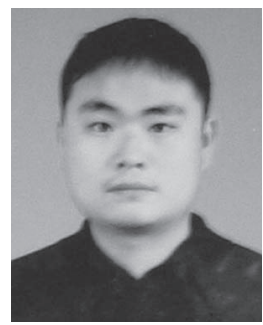

Xinyi Shen (M'11) received the B.S. degree in remote sensing science from Wuhan Univerisity, Wuhan, China, in 2007 and the Ph.D. degree in cartology and geographic information system from Peking University, Beijing, China, in 2012.

$\mathrm{He}$ is currently a Postdoctoral Research Fellow with the Atmospheric Radar Research Center, The University of Oklahoma, Norman, USA. His fields of interest include soil moisture retrieval, microwave remote sensing modeling, and electromagnetic scattering properties of random targets, particularly in the resonant region and photogrammetry.

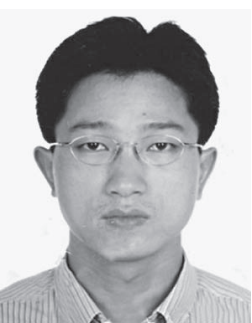

Kebiao Mao received the B.S. degree from Northeast Normal University, Changchun, China, in 2001, the M.S. degree from Nanjing University, Nanjing, China, in 2004, and the Ph.D. degree in geographic information systems from the Chinese Academy of Sciences, Beijing, China, in 2007.

$\mathrm{He}$ is currently with the Key Laboratory of Resources Remote Sensing and Digital Agriculture, Ministry of Agriculture, Institute of Agricultural Resources and Regional Planning, Chinese Academy of Agricultural Sciences, Beijing, as an Associate Professor. He is also an Adjunct Research Professor with the Shaanxi Normal University, Xi' an, China. He has published more than 70 papers in international and Chinese scientific journals and applied for eight patents for inventions. His research interests include global climate change, agricultural disaster, and geophysical parameter retrieval (like land surface temperature and emissivity, soil moisture, and water vapor content)

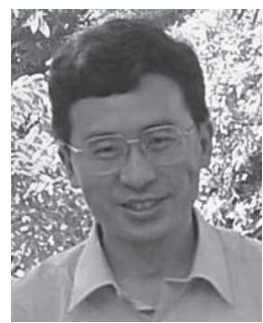

Qiming Qin received the B.S. degree in geography from Nanjing Normal University, Nanjing, China, in 1982, the M.S. degree in geophysics from Shanxi Normal University, Xi'an, China, in 1987, and the $\mathrm{Ph} . \mathrm{D}$. degree in geophysics from Peking University, Beijing, China.

He joined the Mathematical School, Peking University, for a postdoctoral appointment. He is currently a Professor and Vice Dean with the School of Earth and Space Sciences, Peking University. He also serves as the Secretary General of the National Steering Committee for Geography Education in Universities and Colleges, China, and the Remote Sensing Application Consortium of Universities and Colleges, China. He is the Executive Director of The Environmental Remote Sensing Commission of the Geographical Society of China, the Director of China Association for Geographic Information System, Chinese Society for Geodesy, Photogrammetry, and Cartography, China Hi-Tech Industrialization Association, and The Chinese Association of Young Scientists and Technicians. He has served as an evaluator of Domestic GIS software evaluation expert team of China Association for Geographic Information System in 1996 and a lead evaluator of GIS Software Evaluation Committee of The Ministry of Science and Technology of China (1995). 


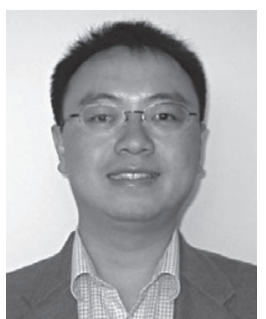

Yang Hong received the B.S. and M.S. degrees in geosciences and environmental sciences from Peking University, Beijing, China, and the Ph.D. degree, major in hydrology and water resources and minor in remote sensing and spatial analysis, from The University of Arizona, Tucson.

Following a postdoctoral appointment with the Center for Hydrometeorology and Remote Sensing, University of California, Irvine, he joined the National Aeronautics and Space Administration Goddard Space Flight Center, Greenbelt, MD, in 2005. $\mathrm{He}$ is currently a Professor with the School of Civil Engineering and Environmental Sciences and the School of Meteorology, The University of Oklahoma, Norman, where he is also directing the Remote Sensing Hydrology research group. He also serves as the Codirector of the WaTER Center and an affiliated Faculty Member with the Atmospheric Radar Research Center. He has served in the editorial boards of the International Journal of Remote Sensing, the Natural Hazards journal, and the Landslides journal. His primary research interests are in remote-sensing retrieval and validation, hydrology and water resources, natural hazard prediction, land surface modeling, and data assimilation systems for water resource planning under changing climate.

Dr. Hong is currently the American Geophysical Union Precipitation Committee Chair.

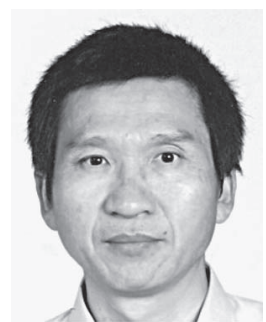

Guifu Zhang (S'97-M'98-SM'02) received the B.S. in physics from Anhui University, Hefei, China, in 1982, the M.S. degree in radio physics from Wuhan University, Wuhan, China, in 1985, and the $\mathrm{Ph} . \mathrm{D}$. degree in electrical engineering from the University of Washington, Seattle, USA, in 1998.

From 1985 to 1993, he was an Assistant and Associate Professor with the Space Physics Department, Wuhan University. In 1989, he worked as a Visiting Scholar with the Communication Research Laboratory, Japan. From 1993 to 1998, he studied and was with the Department of Electrical Engineering, University of Washington, where he first was a Visiting Scientist and later a Ph.D. student. He was a Scientist with the National Center for Atmospheric Research during the period of 1998-2005. After that, he joined the School of Meteorology, The University of Oklahoma, Norman, USA, where he is currently a Professor. He calculated wave scattering from fractal trees, from a target buried under a rough surface. He explored the detection of targets in the presence of clutter using angular correlation. He developed algorithms for retrieving raindrop size distributions. He led to develop advanced signal processing to improve weather radar data quality. He formulated theory of weather radar interferometry and that of phased array radar polarimetry. His research interests include wave propagation and scattering in random and complex media, remote sensing theory and technology for geophysical applications, algorithms for retrieving physical states and processes, cloud and precipitation microphysics and model parameterization, target detection and classification, clutter identification and filtering, radar signal processing, and optimal estimation. He is currently interested in the design and development of polarimetric phased array radars for weather measurements and multimission capability. 